\title{
CONF-960268--5 SAMD96-0373C
}

Operator in-the-loop control of rotary cranes

Gordon G. Parker, Rush D. Robinett, Brian J. Driessen, Clark R. Dohrmann

Sandia National Laboratories

P.O. Box 5800 Mail Stop 0439

Albuquerque, NM 87185-0439

\begin{abstract}
An open-loop control method is presented for reducing the oscillatory motion of rotary crane payloads during operator commanded maneuvers. A typical rotary crane consists of a multiple degree-of-freedom platform for positioning a spherical pendulum with an attached payload. The crane operator positions the payload by issuing a combination of translational and rotational commands to the platform as well as load-line length changes. Frequently, these pendulum modes are time-varying and exhibit low natural frequencies. Maneuvers are therefore performed at rates sufficiently slow so as not to excite oscillation. The strategy presented here generates crane commands which suppress vibration of the payload without a priori knowledge of the desired maneuver. Results are presented for operator in-the-loop positioning using a real-time dynamics simulation of a three-axis rotary crane where the residual sway magnitude is reduced in excess of $40 \mathrm{~dB}$.
\end{abstract}

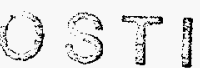

Keywords: Rotary Crane, Control, Swing-Free, Oscillation-Free, Input Shaping

\section{INTRODUCTION}

Construction and transportation cranes can generally be grouped into one of two categories based on their configuration. The first category consists of overhead, gantry cranes. These systems incorporate a trolley which translates in the horizontal plane. Attached to the trolley is a load-line for payload attachment. Typically, they have varying load-line length capabilities. The second category consists of rotary cranes. As the name implies, the load-line attachment point undergoes rotation. Other degrees of freedom may exist such as translation of the load-line attachment point along the jib, variable load-line length, or if the jib is replaced by a boom, the characteristic boom rotational motion, known as luffing ${ }^{*}$.

Auernig and Troger ${ }^{1}$ consider time optimal payload maneuvers of a gantry crane undergoing trolley translation and load-line length change. The coupled, nonlinear equations of motion and adjoint equations, obtained from the application of Pontryagin's maximum principle, are solved analytically for the cases of constant and variable hoisting speeds. In both cases the maneuvers are developed such that the payload is residual oscillation free. Moustafa and Ebeid ${ }^{2}$ demonstrate a state feedback controller for damping load sway for a gantry crane configured to move along two orthogonal directions in the horizontal plane. This work is expanded on by Ebeid et. al. ${ }^{3}$ to incorporate actuator dynamics into the crane model. Fliess et. al. ${ }^{4}$ investigate a feedback linearizing controller for a one-dimensional gantry crane. Trolley traversal and load-line length changes are considered. Simulation results indicate the ability of the closed-loop controller to control load sway for relatively slow maneuvers. This same system is examined by Nguyen ${ }^{5}$ where simulation and experimental results of a nonlinear state-feedback controller is used. Small motions are assumed about a specified operating point. This allows for decoupled equations of motion and decoupled controller design.

Sakawa and Nakazumi ${ }^{6}$ investigate a rotary crane undergoing hub and boom rotation and load hoisting using a combined open and closed-loop approach. The open-loop input to the crane is designed based on a postulated set of functions such that the sway motion of the load is excited minimally. The closed-loop controller is then switched on when the maneuver is near the end, providing significant sway damping. Vaha et. al. ${ }^{7}$ generate suboptimal, minimum time inputs for a rotary crane. Tracking is achieved via a state feedback control law. However, radial sway, due to centripetal acceleration of the payload, is not compensated. Souissi and Koivo ${ }^{8}$ consider a rotary crane undergoing a boom-rotation-boom maneuver using a proportionalintegral-derivative controller similar to Fliess et.al. ${ }^{4}$. The simulation model considers both radial and tangential payload sway, however, the control strategy used results in residual load oscillation. Parker et. al. ${ }^{9}$ investigate residual oscillation free rotary

*Boom rotational motion, referred to as luffing, causes the tip of the boom to move vertically. 
jib crane maneuvers and compare different optimization techniques for generating the crane commands. Knowledge of the initial and final configurations is required in advance.

In this paper the extension of input shaping as described by Feddema ${ }^{10}$ to the nonlinear sway dynamics of a rotary crane are examined. The crane configuration of interest includes one rotational axis (the jib) and two translational axes (trolley position and load-line length). Using configuration dependent input shaping filters, the residual oscillations of the payload are significantly reduced for general, simultaneous three-axis maneuvers. Test results are presented where the nonlinear sway dynamics are simulated in real-time using a digital signal processing (DSP) board. The three axis commands are supplied by the operator via two analog joysticks.

\section{CRANE DESCRIPTION}

The rotary crane considered consists of a translating load-line and payload assembly attached to a translating trolley. The trolley rides along a rotating jib. This apparatus is shown in Fig. 1.

The $\hat{a}_{1}, \hat{a}_{2}, \hat{a}_{3}$ coordinate system is attached to the jib and rotates about the hub with angular rate $\dot{\gamma}(t)$. The rotation angles $\theta(t), \phi(t)$ are defined as rotations of the load-line about the $\hat{a}_{1}, \hat{a}_{2}$ axes respectively. The attachment point of the load-line to the jib is at a distance $x(t)$ from the center of rotation of the hub. The load-line has length $L(t)$ and the payload has mass $m$. The two payload sway rotations will from now on be referred to as the radial $(\phi)$ and the tangential $(\theta)$. The inputs to this system are the output of velocity tracking servo controllers at each axis. Assuming perfect tracking, which is the worst case for inducing residual vibration to the payload, the inputs are simply $\dot{\gamma}, \dot{x}, \dot{L}$. The outputs are the rotation angles of the load-line, $\theta(t), \phi(t)$

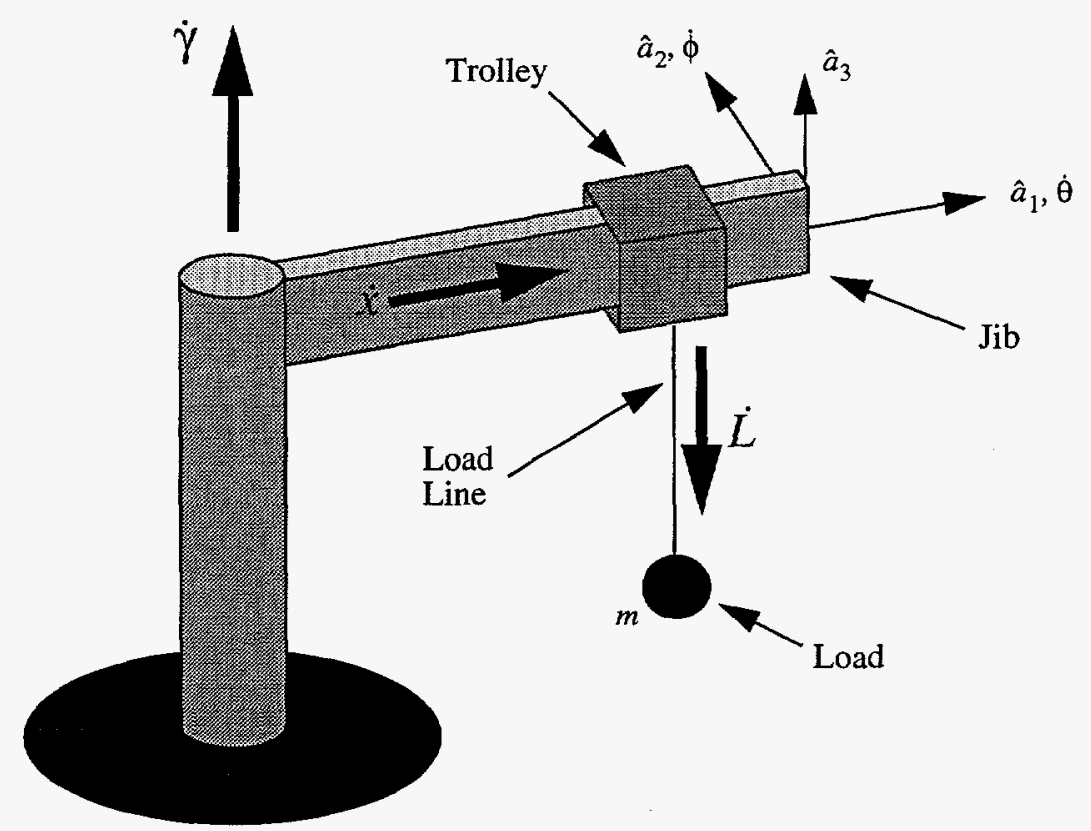

Figure 1. A Rotary Crane. 


\section{EQUATIONS OF MOTION}

Using the rotating $\hat{a}_{1}, \hat{a}_{2}, \hat{a}_{3}$ coordinate system, the vector from the center of rotation of the hub to the payload is given by

$$
\begin{aligned}
\underline{p}(t)= & (x(t)-L(t) \cos [\theta(t)] \sin [\phi(t)]) \hat{a}_{1}+ \\
& L(t) \sin [\theta(t)] \hat{a}_{2}- \\
& L(t) \cos [\theta(t)] \cos [\phi(t)] \hat{a}_{3}
\end{aligned}
$$

Lagrange's equations

$$
\frac{d}{d t}\left(\frac{\partial \mathcal{L}}{\partial \dot{q}_{i}}\right)-\frac{\partial \mathcal{L}}{\partial q_{i}}=0
$$

with $q_{1}=\theta$ and $q_{2}=\phi$ are applied to this system where the Lagrangian $\mathcal{L}$

$$
\mathcal{L}=T-V
$$

is the difference between the kinetic energy $T$ and the potential energy $V$.

With the aid of Fig. 1 and Eq. 1, the kinetic energy can be found by evaluating

$$
\begin{gathered}
T=\frac{1}{2} m \dot{\underline{L}}^{T} \underline{\dot{p}} \\
\dot{\underline{p}}=[\dot{x}-\dot{L} \cos \theta \sin \phi+L \dot{\theta} \sin \theta \sin \phi-L \dot{\phi} \cos \theta \cos \phi-L \dot{\gamma} \sin \theta] \hat{a}_{1}+ \\
{[\dot{L} \sin \theta+L \dot{\theta} \cos \theta+\dot{\gamma}(x-L \cos \theta \sin \phi)] \hat{a}_{2}+} \\
{[-\dot{L} \cos \theta \cos \phi+L \dot{\theta} \sin \theta \cos \phi+L \dot{\phi} \cos \theta \sin \phi] \hat{a}_{3}}
\end{gathered}
$$

where $\underline{\dot{p}}$ is the absolute velocity of the payload in the rotating coordinate frame.

The potential energy

$$
V=-m g L \cos \phi \cos \theta
$$

is also obtained from examining Fig. 1.

Substituting Eq. 3, through Eq. 6 into Eq. 2 yields the two equations of motion for the oscillatory degrees of freedom $\phi, \theta$

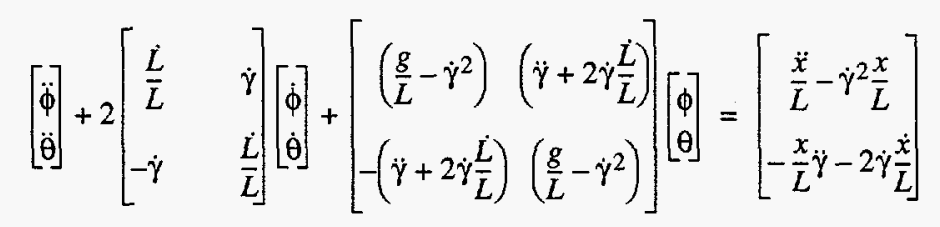

where small angle assumptions have been exploited for $\phi, \theta$ and their derivatives. Note that the quantities $\gamma$, and $L$ are functions of time, resulting in a time-varying stiffness effect. Detailed discussion of this phenomenon for related systems is discussed by Petterson and Robinett ${ }^{11}$. 


\section{FILTER GENERATION}

In this section the procedure for designing forward path command filters for the jib rotation $(\dot{\gamma})$ and the trolley translation $(\dot{x})$ is described. Using a simplified model of Eq. 7, the system is transformed to modal coordinates. Decoupled forward path filters are designed in the modal coordinate system with configuration dependent, imaginary coefficients. After transforming back to the physical coordinates, the filters between $\dot{\gamma}$ and $\dot{x}$ become coupled, with configuration dependent, real coefficients.

To simplify the model of Eq. 7 further, we will assume that $\dot{x}, \dot{L}$, and $\dot{\gamma}$ are of the same order of magnitude as $\dot{\theta}$ and $\dot{\phi}$. The resulting simplified model

$$
\left[\begin{array}{l}
\ddot{\phi} \\
\ddot{\theta}
\end{array}\right]+\left[\begin{array}{cc}
\frac{g}{L} & \ddot{\gamma} \\
-\ddot{\gamma} & \frac{g}{L}
\end{array}\right]\left[\begin{array}{l}
\phi \\
\theta
\end{array}\right]=\left[\begin{array}{c}
\ddot{x} \\
L \\
-\frac{x}{L}
\end{array}\right]
$$

is obtained by retaining terms of order less than two. This system may be written symbolically as

$$
M \ddot{\Phi}+K \underline{\Phi}=\underline{u}
$$

where the matrix $M$ is a $2 \times 2$ identity matrix, $K$ is a $2 \times 2$ matrix chosen appropriately from Eq. 8 and $\underline{q}$ is a $2 \times 1$ vector of the terms on the right side of Eq. 8. Eigenvalues and eigenvectors are found by solving

$$
\left(K-\omega^{2} M\right) \underline{e}=0
$$

yielding

$$
\omega_{1,2}^{2}=\frac{g}{L} \pm \ddot{\gamma} i
$$

and

$$
E=\left[\begin{array}{ll}
e_{1}^{T} & e_{2}^{T}
\end{array}\right]=\left[\begin{array}{cc}
1 & i \\
-i & 1
\end{array}\right]
$$

where $i$ is the square root of -1 .

The system of Eq. 9 can be transformed to

$$
I \underline{\ddot{y}}+\Omega \underline{y}=\underline{U}
$$

where

$$
\begin{gathered}
\Phi=E\left[\begin{array}{l}
y_{1} \\
y_{2}
\end{array}\right]=E \underline{y} \\
{\left[\begin{array}{l}
U_{1} \\
U_{2}
\end{array}\right]=\underline{U}=E^{-1} \underline{\underline{u}}}
\end{gathered}
$$

and 


$$
\Omega=E^{-1} K E=\left[\begin{array}{cc}
\frac{g}{L}+\ddot{\gamma} i & 0 \\
0 & \frac{g}{L}-\ddot{\gamma} i
\end{array}\right]
$$

Now, the forward path filters are designed in the modal coordinate space after representing the decoupled system of Eq. 13 in the Laplace domain

$$
\frac{y_{1}}{U_{1}}=\frac{1}{s^{2}+\left(\frac{g}{L}+\ddot{\gamma} i\right)} \quad \frac{y_{2}}{U_{2}}=\frac{1}{s^{2}+\left(\frac{g}{L}-\ddot{\gamma} i\right)}
$$

requiring that $\ddot{\gamma}$ and $L$ are constant over each finite length sample period. The filtered inputs, designated as $\underline{U}_{F}$ are

$$
\underline{U}_{F}=B \underline{U}
$$

where the $2 \times 2$ matrix $B$ is

$$
B=\left[\begin{array}{cc}
\frac{K_{1}\left[s^{2}+\left(\frac{g}{L}+i \ddot{\gamma}\right)\right]}{\left(s+a_{1}\right)^{3}} & 0 \\
0 & \frac{K_{2}\left[s^{2}+\left(\frac{g}{L}-i \ddot{\gamma}\right)\right]}{\left(s+a_{2}\right)^{3}}
\end{array}\right]
$$

where

$$
K_{1}=\frac{a_{1}^{3}}{\frac{g}{L}+i \ddot{\gamma}} \quad K_{2}=\frac{a_{2}^{3}}{\frac{g}{L}-i \ddot{\gamma}}
$$

are chosen to yield unity steady-state gain of the filtered inputs. These transfer functions in $B$ approximate configuration dependent notch filters, where the design parameters $a_{1}$ and $a_{2}$ determine the amount of roll-off after the notch.

The filtered inputs in physical coordinates are then

$$
\begin{gathered}
\underline{\mathcal{U}}_{\mathscr{F}}=E \underline{U}_{F}=E B \underline{U}=E B E^{-1} \underline{\underline{U}} \\
\underline{\underline{U}}_{\mathscr{F}}=\frac{1}{2}\left[\begin{array}{cc}
B_{11}+B_{22} & i\left(B_{22}-B_{11}\right) \\
-i\left(B_{22}-B_{11}\right) & B_{11}+B_{22}
\end{array}\right] \underline{\underline{U}}
\end{gathered}
$$

The expressions for $B_{11}+B_{22}$ and $B_{22}-B_{11}$ are greatly simplified by enforcing the design constraint of $a=a_{1}=a_{2}$ resulting in an explicit representation of Eq. 20 as 


$$
\underline{u}_{\mathcal{F}}=\left[\begin{array}{cc}
\frac{a^{3} \beta\left(s^{2}+\frac{1}{\beta}\right)}{(s+a)^{3}} & \frac{-\Gamma a^{3} s^{2}}{(s+a)^{3}} \\
\frac{\Gamma a^{3} s^{2}}{(s+a)^{3}} & \frac{a^{3} \beta\left(s^{2}+\frac{1}{\beta}\right)}{(s+a)^{3}}
\end{array}\right] \underline{\underline{U}}
$$

where

$$
\beta=\frac{g}{L\left[\left(\frac{g}{L}\right)^{2}+\ddot{\gamma}^{2}\right]} \quad \Gamma=\frac{\ddot{\gamma}}{\left(\frac{g}{L}\right)^{2}+\ddot{\gamma}^{2}}
$$

The filters of Eq. 21 operate on the inputs as defined by Eq. 8 and Eq. 9, or, individually they may be expressed as

$$
\begin{aligned}
& u_{1_{J}}=\frac{a^{3} \beta\left(s^{2}+\frac{1}{\beta}\right)}{L(s+a)^{3}}[\ddot{x}]+\frac{\Gamma a^{3} s^{2}}{L(s+a)^{3}}[x \dot{\gamma}] \\
& u_{2_{g}}=\frac{\Gamma a^{3} s^{2}}{L(s+a)^{3}}[\ddot{x}]-\frac{a^{3} \beta\left(s^{2}+\frac{1}{\beta}\right)}{L(s+a)^{3}}[x \ddot{\gamma}]
\end{aligned}
$$

The filtered inputs to the sway dynamics of Eq. 7 become

$$
\begin{gathered}
\ddot{x}_{F}=\frac{a^{3} \beta\left(s^{2}+\frac{1}{\beta}\right)}{(s+a)^{3}}[\ddot{x}]+\frac{\Gamma a^{3} s^{2}}{(s+a)^{3}}[x \ddot{\gamma}] \\
\ddot{\gamma}_{F}=\frac{\left(\Gamma a^{3} s^{2}\right)}{x(s+a)^{3}}[\ddot{x}]-\frac{a^{3} \beta\left(s^{2}+\frac{1}{\beta}\right)}{x(s+a)^{3}}[x \ddot{\gamma}]
\end{gathered}
$$

where, of course, $\ddot{x}$ and $\ddot{\gamma}$ are the commanded quantities. Note, that if the jib angular acceleration is significantly less than the static pendulum natural frequency $\sqrt{g / L}$, then from Eq. 22, $\Gamma$ is approximately zero. The filters of Eq. 24 are then decoupled.

\section{OPERATOR IN-THE-LOOP SIMULATION}

A real-time, operator in-the-loop crane simulator is used to test the forward path filters described above. The simulator allows the operator to enter velocity commands in the three-axes using two joysticks. Visual cues of the crane motion and sway properties are supplied via a graphical output of the results of a real-time simulation of the crane sway dynamics. This system is intended for use not only as a control algorithm testbed, but also as a crane operator training device. The computational portion of the system uses a dSPACE platform. Specifically, a DSP board (DS3001) is used for performing the sway simulation and for filter implementation, both running at $100 \mathrm{~Hz}$. Two Gravis analog joysticks are connected to a multi-I/O board (DS2201) and are sampled at $10 \mathrm{~Hz}$. Operator feedback of payload motion and sway dynamics is provided by a graphical user interface (COCKPIT). For comparison purposes, the unfiltered crane acceleration inputs are simultaneously passed to a duplicate version of the crane sway dynamics.

Since the jib rotation acceleration is typically well below $\sqrt{g / L}$, the decoupled version of the filters of Eq. 24 was used as well as the approximation of $\beta$ as $\beta \approx L / g$. Load-line length actuator dynamics were approximated by a $0.2 \mathrm{~Hz}$ low pass filter on load-line length velocity commands. Furthermore, it was found that the filtering of the quantity $x \dot{\gamma}$ for the $\ddot{\gamma}_{F}$ filter of Eq. 24 
results in a necessary operator correction for residual velocity due to a lack of synchronization between the filtered and unfiltered trolley position, expressed compactly by

$$
\frac{[x \ddot{\gamma}]_{F}}{x} \neq \ddot{\gamma}_{F}
$$

The filter design was performed by assuming that the trolley position was constant over each finite length sample period. The resulting filters are

$$
\ddot{x}_{F}=\frac{a^{3} \frac{L}{g}\left(s^{2}+\frac{g}{L}\right)}{(s+a)^{3}}[\ddot{x}] \quad \quad \ddot{\gamma}_{F}=\frac{a^{3} \frac{L}{g}\left(s^{2}+\frac{g}{L}\right)}{(s+a)^{3}}[\ddot{\gamma}]
$$

where the single design parameter, $a$, was chosen as 2.0 . The filters were implemented by converting the continuous time, state space representation to discrete time ${ }^{12}$.

The results presented start with the load-line length initially at $0.25 \mathrm{~m}$ (a $1 \mathrm{~Hz}$ nonrotating natural frequency), the trolley position is at $0.5 \mathrm{~m}$ and the jib angle is at $0^{\circ}$. Data is recorded at $10 \mathrm{~Hz}$ for an arbitrary set of stick commands over a 40 second period. Plots of the jib, trolley and load-line motion are shown in Fig. 2 through Fig. 4 . The noticeable lag between filtered and unfiltered motion in the jib rotation and trolley position is due to the notch filtering discussed previously. The configuration dependent notch filtering yields a decrease in the residual oscillation magnitude of $43 \mathrm{~dB}$ in the tangential direction and a decrease of $47 \mathrm{~dB}$ in the radial as shown by the tangential and radial sway histories of Fig. 5 and Fig. 6.

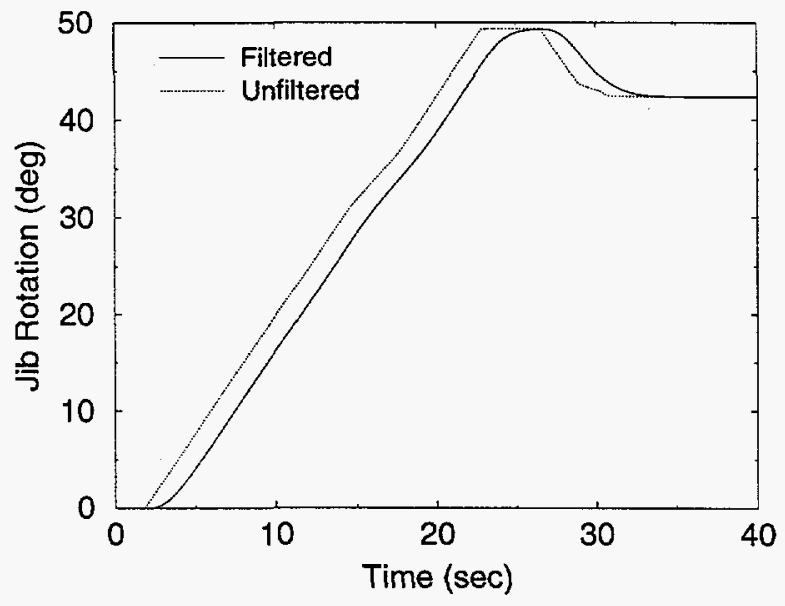

Figure 2. Jib rotation time history for filtered and unfiltered input commands.

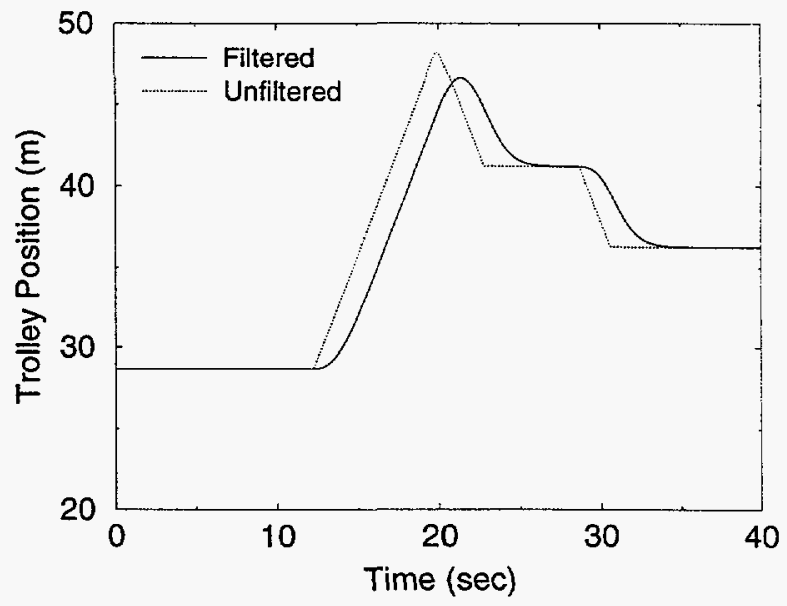

Figure 3. Trolley position time history for filtered and unfiltered input commands. 


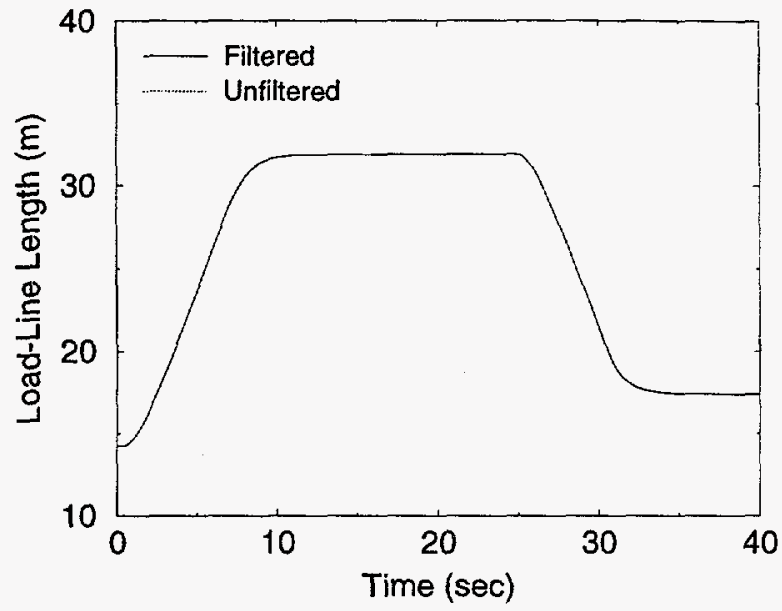

Figure 4. Load-line length time history for filtered input commands.

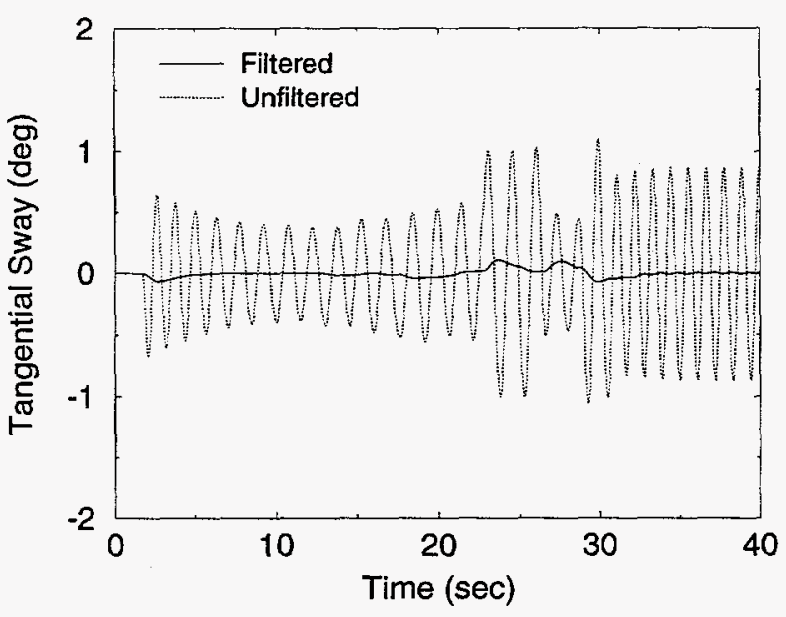

Figure 5. Tangential payload sway for filtered and unfiltered inputs

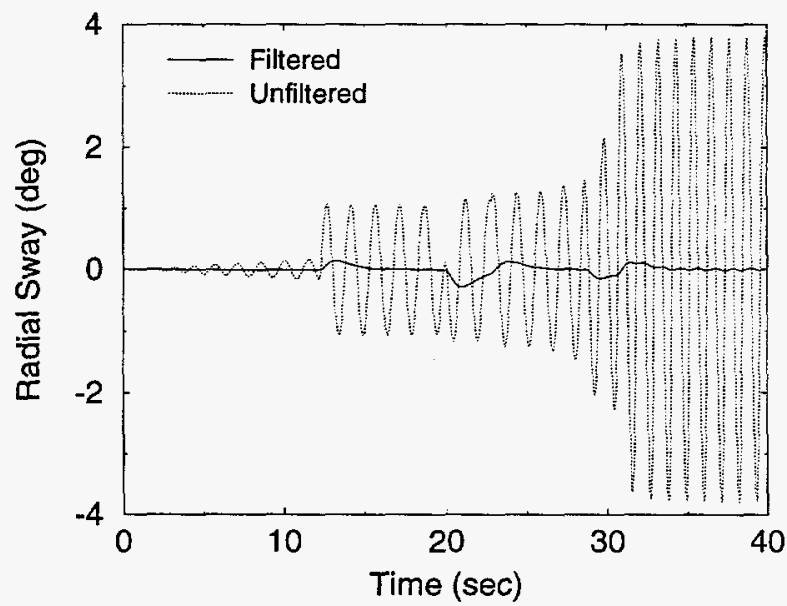

Figure 6. Radial payload sway for filtered and unfiltered inputs

\section{SUMMARY}

A method for reducing the residual oscillation of operator in-the-loop point-to-point rotary crane maneuvers was presented. Testing was performed using an operator in-the-loop crane simulator designed as a control algorithm testbed and a crane operator training device. Configuration dependent notch filters were proposed for filtering forward path velocity commands of the jib rotation speed and trolley speed. Operator in-the-loop, real-time simulation indicates a decrease in the residual oscillation magnitude in excess of $40 \mathrm{~dB}$ in both the tangential and radial directions. Unlike the optimization based methods ${ }^{9}$, the forward path filter design does not require a priori knowledge of the specific maneuver of interest.

\section{ACKNOWLEDGMENTS}

This work performed at Sandia National Laboratories is supported by the U.S. Department of Energy under contract DE-AC0494AL85000. 


\section{REFERENCES}

1. J.W. Auernig and H. Troger, "Time Optimal Control of Overhead Cranes with Hoisting of the Load," Automatica, Vol. 23, No. 4, pp. 437-447, 1987.

2. K.A.F. Moustafa and A.M. Ebeid, "Nonlinear Modeling and Control of Overhead Crane Load Sway," Journal of Dynamic Systems, Measurement, and Control, Vol. 110, pp. 266-271, 1988.

3. A.M. Ebeid, K.A.F. Moustafa and H.E. Emara-Shabaik, "Electromechanical Modelling of Overhead Cranes," International Journal of Systems Science, Vol. 23, No. 12, pp. 2155-2169, 1992.

4. M. Fliess, J. Levine P. Rouchon, "A Simplified Approach of Crane Control via a Generalized State-Space Model," IEEE Proceedings of the 30th Conference on Decision and Control, Brighton, England, pp. 736-741, 1991.

5. H.T. Nguyen, "State-Variable Feedback Controller for an Overhead Crane," Journal of Electrical and Electronics Engineering, Australia, Vol. 14, No. 2, pp. 75-84, 1994.

6. Y. Sakawa and A. Nakazumi, "Modeling and Control of a Rotary Crane," Journal of Dynamic Systems, Measurement, and Control, Vol. 107, pp. 200-206, 1985.

7. P. Vaha, S. Pieska and E. Timonen, "Robotization of an Offshore Container Crane," Robots: Coming of Age, Proceedings of the 19th ISIR International Symposium, pp. 637-648, 1988.

8. R. Souissi and A.J. Koivo, "Modeling and Control of a Rotary Crane for Swing-Free Transport of Payloads," The First IEEE Conference on Control Applications, Dayton, OH, pp. 782-787, 1992.

9. Parker, G.G., Petterson, B., Dohrmann, C.R. and Robinett, R.D., "Vibration Suppression of Fixed-Time Jib Crane Maneuvers," Proceedings of the SPIE Smart Structures and Materials Conference, pp. 131-140, 1995.

10. Feddema J.T., "Digital Filter Control of Remotely Operated Flexible Robotic Structures," Proceedings of the 1993 American Control Conference.

11. B.J. Petterson and R.D. Robinett, "Model-Based Damping of Coupled Horizontal and Vertical Oscillations in a Flexible Rod," Journal of Intelligent and Robotic Systems, Vol. 4, pp. 285-299, 1991.

12. C.T. Chen, "Linear System Theory and Design", Holt, Rinehart and Winston, New York, NY, 1984.

\section{DISCLAIMER}

\footnotetext{
This report was prepared as an account of work sponsored by an agency of the United States Government. Neither the United States Government nor any agency thereof, nor any of their employees, makes any warranty, express or implied, or assumes any legal liability or responsibility for the accuracy, completeness, or usefulness of any information, apparatus, product, or process disclosed, or represents that its use would not infringe privately owned rights. Refermanufacturer, ary specific commercial product, process, or service by trade name, trademark, manufacturer, or otherwise does not necessarily constitute or imply its endorsement, recommendation, or favoring by the United States Government or any agency thereof. The views and opinions of authors expressed herein do not necessarily state or reflect those of the United States Government or any agency thereof.
} 


\section{DISCLAMMIER}

Portions of this document may be illegible in electronic image products. Images are produced from the best available original document. 